\title{
Suppression of Discharge Current Oscillations in a Hall Thruster*
}

\author{
By Naoji Yамамото, ${ }^{1)}$ Shigeru YoкотA, ${ }^{2)}$ Keiko Watanabe, ${ }^{3)}$ \\ Akihiro SASOH, ${ }^{3)}$ Kimiya KomURASAKI ${ }^{4)}$ and Yoshihiro ARAKawa ${ }^{2)}$ \\ ${ }^{1)}$ Department of Advanced Energy Engineering Science, Kyushu University, Fukuoka, Japan \\ ${ }^{2)}$ Department of Aeronautics and Astronautics, The University of Tokyo, Tokyo, Japan \\ ${ }^{3)}$ Institute of Fluid Science, Tohoku University, Sendai, Japan \\ ${ }^{4)}$ Department of Advanced Energy, The University of Tokyo, Kashiwa, Japan
}

(Received March 10th, 2005)

Results of controlling a discharge current oscillation in Hall thrusters at a frequency range of $10-100 \mathrm{kHz}$ are presented. To understand the discharge current oscillation mechanism, the plasma behavior in the acceleration channel was observed with a high-speed camera using a 1-kW class, anode layer type Hall thruster. The emission intensity oscillates equably in the acceleration channel at the same period of the discharge current oscillation; the number density of excited xenon ions oscillates at the same oscillation period and is proportional to the discharge current. These results indicate that the discharge current oscillation is caused by the ionization instability and the number density of plasma oscillates equably in the acceleration channel. Furthermore, the oscillation amplitude was sensitive to the applied magnetic flux density, indicating that this oscillation is affected by electron mobility. The proposed oscillation model based on the experimental results demonstrated that the momentum transfer corresponding to a plasma fluctuation is crucial to achieving stability. Thus, the oscillation amplitude for various acceleration channel configurations-parallel and convergent-was measured, because channel configuration could affect the momentum transfer. The oscillation was successfully suppressed by adopting the convergent configuration, as shown by this model.

Key Words: $\quad$ Electric Propulsion, Hall Thruster, Discharge Oscillation, High-speed Camera

\author{
Nomenclature \\ $B$ : magnetic flux density \\ $D$ : diffusion coefficient \\ $E$ : electric field strength \\ $e$ : electronic charge \\ $I_{\mathrm{d}}$ : discharge current \\ $k$ : wave number \\ $L$ : ionization zone length \\ $l$ : length \\ $m$ : particle mass \\ $\dot{m}$ : mass flow rate \\ $N$ : number density \\ $S$ : cross-section \\ $T$ : temperature \\ $V$ : volume \\ $V_{\mathrm{e}}$ : electron velocity \\ $V_{\mathrm{n}}$ : neutral atom velocity \\ $V_{\mathrm{d}}$ : discharge voltage \\ $z:$ axial direction \\ $\phi$ : diameter \\ $\lambda_{\text {ne }}$ : neutral-electron mean free path \\ $\mu$ : mobility \\ $\sigma_{\mathrm{di}}:$ atom ionization collision cross section \\ $\sigma_{\mathrm{T}}$ : atom total collision cross section \\ $\tau$ : measurement time \\ $\omega$ : oscillation frequency
}

(C) 2005 The Japan Society for Aeronautical and Space Sciences

*Presented at ISTS on May 2004.

\author{
Subscripts \\ e: electron \\ i: ion \\ $\mathrm{n}$ : neutral atom \\ 0: anode side \\ 1: exit side
}

\section{Introduction}

A Hall thruster is a promising thruster for satellite station keeping and orbit transfer applications ${ }^{1,2)}$ because it has a high thrust efficiency exceeding $50 \%$ with a specific impulse range of 1,000-3,000 s, delivering higher thrust-to-power ratio as compared to ion thrusters. There are several types of Hall thrusters and they can be categorized into two general groups: magnetic layer type and anode layer type. ${ }^{3,4)}$ An example of the former type is the Stationary Plasma Thruster (SPT) developed in Russia. ${ }^{5}$ ) The distinguishing feature of this type is continuous and extended acceleration zones for sufficient ionization and stability. It has a ceramic wall and the length of the acceleration channel is greater than its channel width. ${ }^{6)}$ On the other hand, the Thruster with Anode Layer (TAL) also developed in Russia ${ }^{7-9)}$ is an example of the anode layer type. This thruster has a narrow acceleration zone for reducing loss caused by ion and electron collisions with the walls. It has a conducting wall whose potential is maintained at the same value as the cathode potential and its acceleration channel length is shorter than its channel width. ${ }^{10,11)}$ The short acceleration length and the 
high-resistive material give the anode layer type a potential longer lifespan than observed SPT's. ${ }^{12)}$ However, it can operate in a very limited range of operational conditions compared to SPT's, so it has been backward compared with the other in practical applications.

One problem of Hall thrusters relates to the discharge current oscillation, particularly at a frequency range of 10$100 \mathrm{kHz}$. It is preferable to maintain a low level of discharge current oscillations due to a reduction in the impact of the power processing unit (PPU) and an increase in the margin of the satellite power supply. The oscillation may also increase erosion of the acceleration channel or ion beam divergence. Understanding the oscillation is essential for future improvement of Hall thrusters-particularly the anode layer type. Several studies conducted on the oscillation phenomenon have revealed that this oscillation is caused by ionization instability. ${ }^{13-19)}$ However, these studies did not adequately describe this oscillation-especially the stability criteria for a given range of the magnetic flux density. This study aims to investigate plasma behavior in the acceleration channel using a high-speed camera and propose a physical model of the oscillation based on experimental results in order to suppress the discharge current oscillation.

\section{Experimental Equipment}

\subsection{Thruster}

Figure 1 shows the cross section of a 1-kW class, anode layer type Hall thruster. ${ }^{20)}$ The inner and outer diameter of the acceleration channel are $48 \mathrm{~mm}$ and $72 \mathrm{~mm}$, respectively. A solenoidal coil at the center of the thruster applies a radial magnetic field in the acceleration channel. The magnetic flux density is varied by changing the coil current. There is no outer coil because a uniform magnetic field distribution is maintained along the azimuthal direction. The magnetic field distribution along the channel median is almost uni-

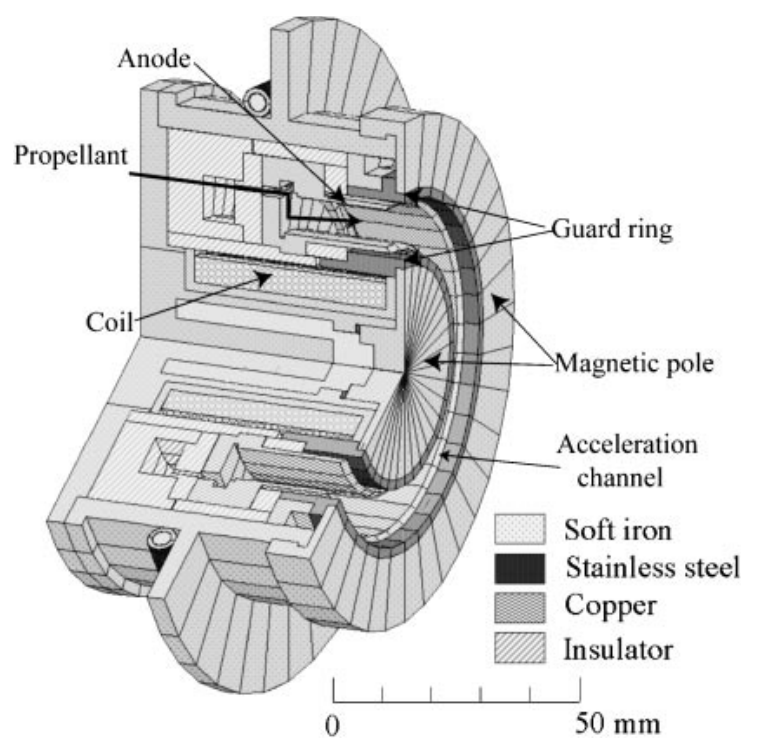

Fig. 1. Cross-section of the Hall Thruster developed at University of Tokyo.

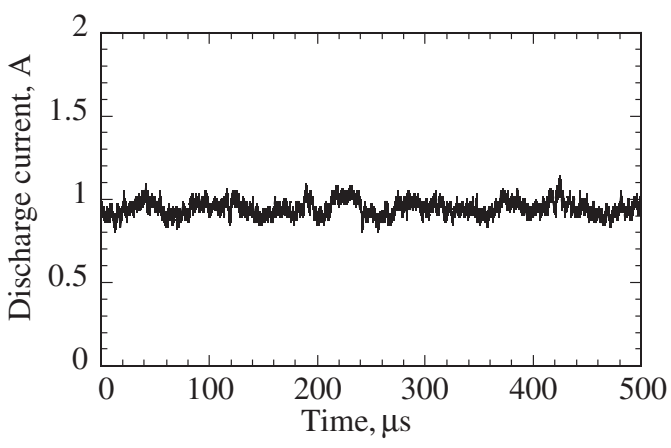

Fig. 2. Discharge current trace of hollow cathode.

form in the short acceleration channel. The magnetic flux density is maximized at the inner wall and reduces with increasing radius because the magnetic flux is constant. Thus, this study assumes the magnetic flux density in the channel median to be representative. The thruster has a hollow annular anode comprising two cylindrical rings through which xenon propellant gas is fed. The gap between the tip of the anode and the exit of the acceleration channel is fixed at $3 \mathrm{~mm}$. A filament cathode $(\phi=0.27 \mathrm{~mm}, l=400 \mathrm{~mm} \times$ $3,2 \%$ thoriated tungsten) was used as the electron source, because a hollow cathode can be a noise source in itself (Fig. 2).

\subsection{Vacuum chamber}

A vacuum chamber- $2 \mathrm{~m}$ in diameter and $3 \mathrm{~m}$ in lengthwas used in the experiments. The pumping system comprised a diffusion pump, a mechanical booster pump and two rotary pumps. The background pressure was maintained below $5.3 \times 10^{-3} \mathrm{~Pa}$ for most of the operating conditions.

\subsection{High-speed camera}

An image intensified high-speed camera (DRS Technologies Inc, ULTRA 8) was used to observe the plasma. The maximum frame rate of this camera is one hundred million frames per second.

\section{Results and Discussion}

\subsection{Oscillation characteristics}

To evaluate the oscillation depth in the experimental results, the amplitude of oscillation $\Delta$ is defined as:

$$
\Delta=\frac{\text { R.M.S }}{\overline{I_{\mathrm{d}}}}=\frac{1}{\overline{I_{\mathrm{d}}}} \sqrt{\frac{\int_{0}^{\tau}\left(I_{\mathrm{d}}-\overline{I_{\mathrm{d}}}\right)^{2}}{\tau}}, \quad\left(\overline{I_{\mathrm{d}}}=\frac{\int_{0}^{\tau} I_{\mathrm{d}}}{\tau}\right)
$$

Figure 3 shows the relationship between $\Delta$ and $B$. $\Delta$ was found to be sensitive to $B$. Stable operation is observed in a very narrow range near $B \approx 16 \mathrm{mT}$ or $B>27 \mathrm{mT}$, although the thrust efficiency is low for $B>27 \mathrm{mT}$. This sensitivity to $B$ indicates that the oscillation is affected by electron mobility rather than by ion mobility.

To observe the events in the acceleration channel, the plasma behavior was observed using the high-speed camera. Figure 4(a) shows the plasma behavior and discharge current under an unstable condition at a frame rate of $350,000 \mathrm{fps}$ and an exposure time of $1 \mu \mathrm{s}$. Intense emission 


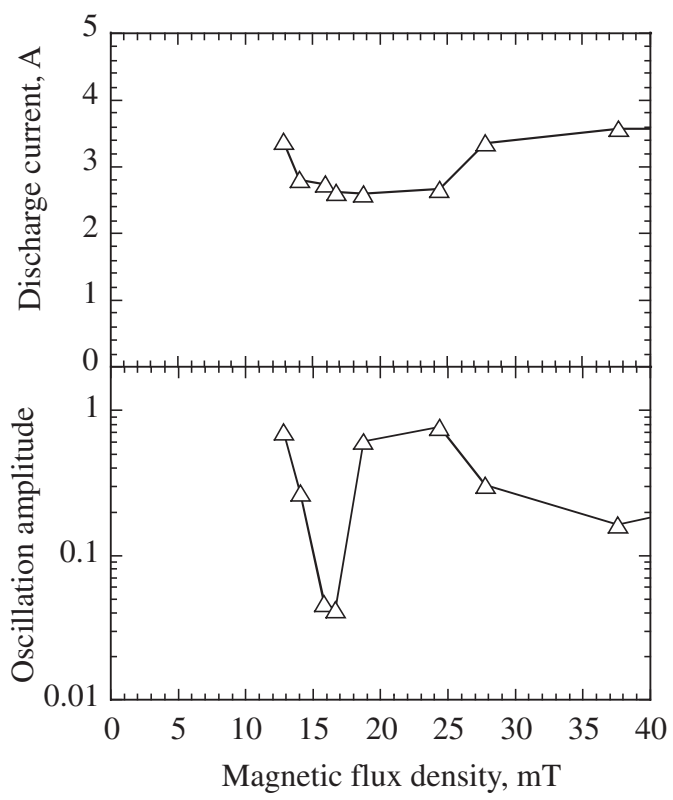

Fig. 3. Oscillation characteristics.

is observed in the annular acceleration channel and it oscillates equably in the channel with the same period as that of the discharge current oscillation. The intensity is approximately proportional to $I_{\mathrm{d}}$ and there is a period when the emission intensity was almost zero. On the other hand, the emission intensity during stable operation was nearly con- stant (Fig. 4(b)). The emission intensity provides significant information on ionization because it is proportional to the number density of the excited species, which is related to the number density of the plasma. To detect the particular spectrum (Xe II, $460.3 \mathrm{~nm}, 5 \mathrm{p}^{4} 6 \mathrm{p}^{4} \mathrm{D}_{3 / 2} \rightarrow 5 \mathrm{p}^{4} 6 \mathrm{~s}^{4} \mathrm{P}_{3 / 2}$ ), the emission was observed through a band-pass filter (center wavelength $=456 \mathrm{~nm}$, full width at half-maximum $=3$ $\mathrm{nm})$. Figure 5 shows the behavior of the xenon ions in the excited state. The frame rate was $500,000 \mathrm{fps}$ and the exposure time was $1 \mu \mathrm{s}$. The number density of $5 p^{4} 6 p^{4} \mathrm{D}_{3 / 2}$ oscillates and is proportional to $I_{\mathrm{d}}$ (Fig. 4(a)).

These results show that the oscillation is derived from the plasma density fluctuation in the acceleration channel, i.e., this oscillation is caused by ionization instability. In addition, the plasma in the annular acceleration channel oscillates monolithically in the entire ionization zone.

\subsection{Oscillation model}

Several studies have shown that the discharge current oscillation is caused by ionization instability, i.e., a disturbance in the number density of neutral atoms causes a disturbance in the plasma density, and it feeds back to the disturbance in the neutral density. There is a time lag between performing the neutral atom feed and plasma feed, so the number density in each particle continues to oscillate without convergence.

The equations describing this oscillation consist of the equation of continuity for the neutral atom and the equation
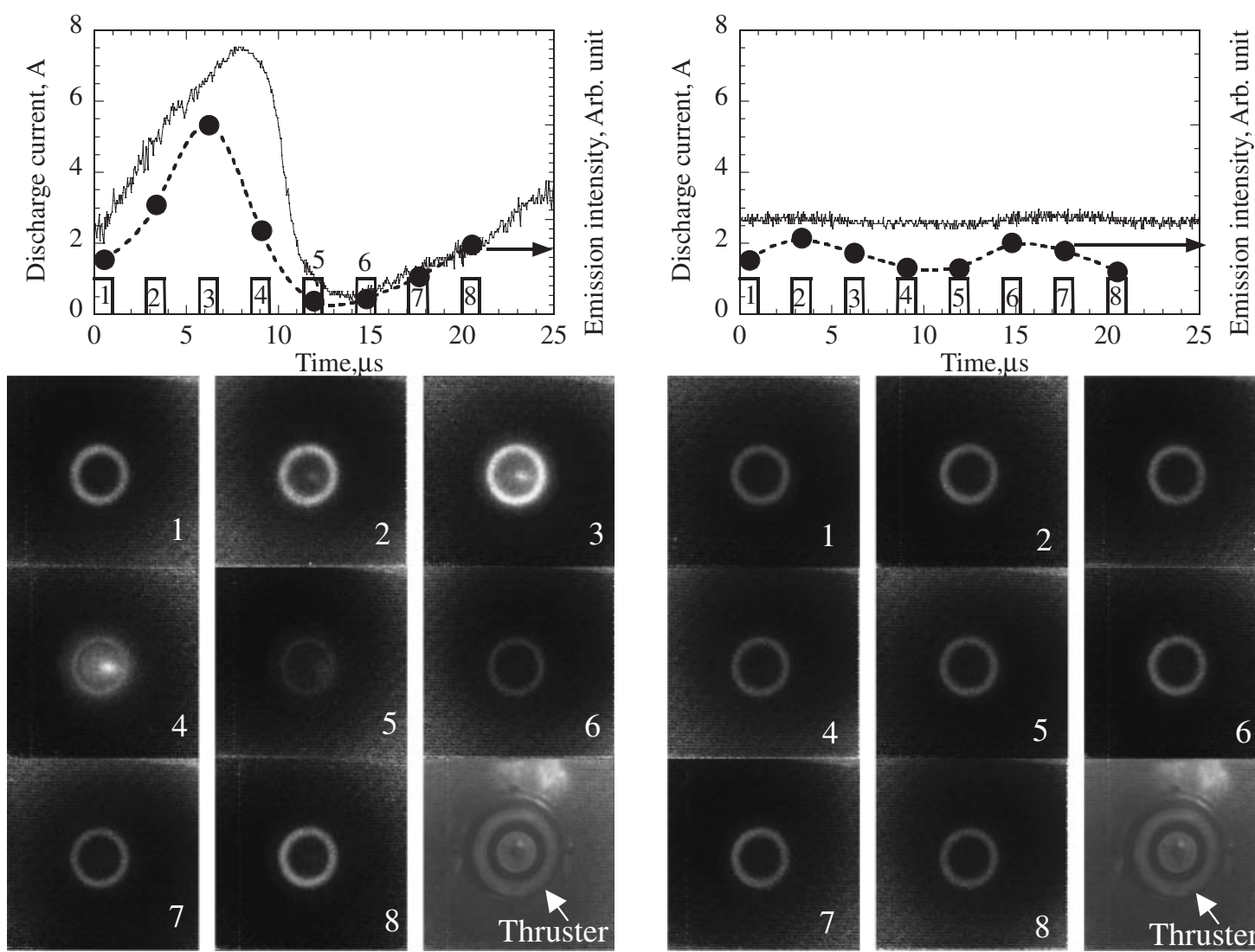

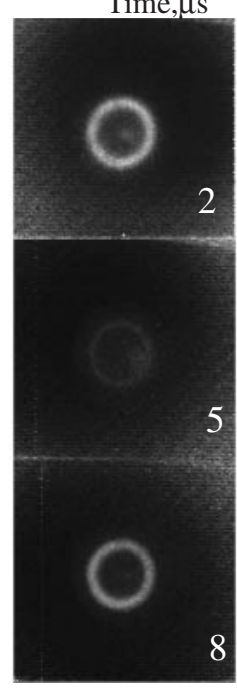

(a)

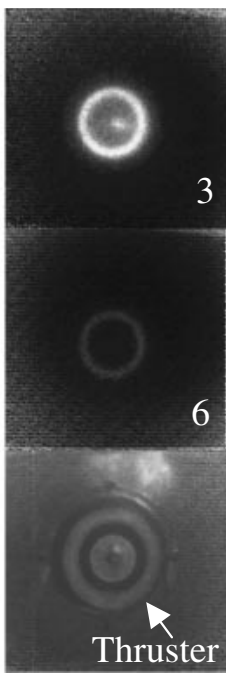

6
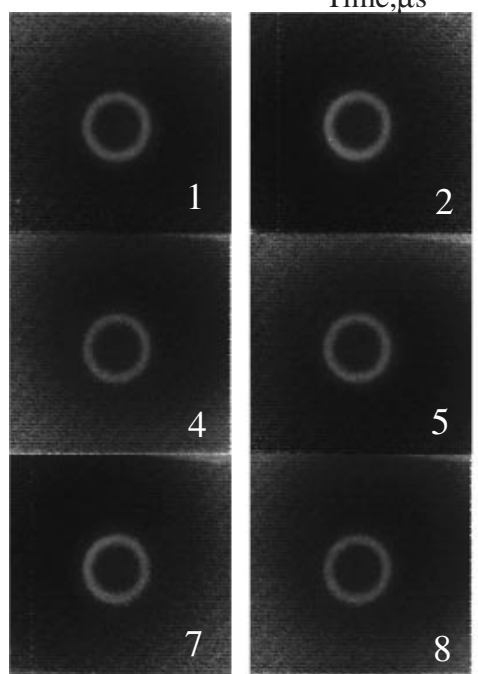

(b)

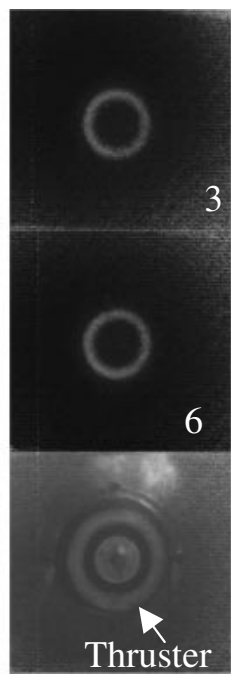

6

Fig. 4. Oscillation behavior of plasma.

(a) unstable condition, $B_{\mathrm{r}}=25 \mathrm{mT}$. (b) stable condition, $B_{\mathrm{r}}=16 \mathrm{mT}$. 

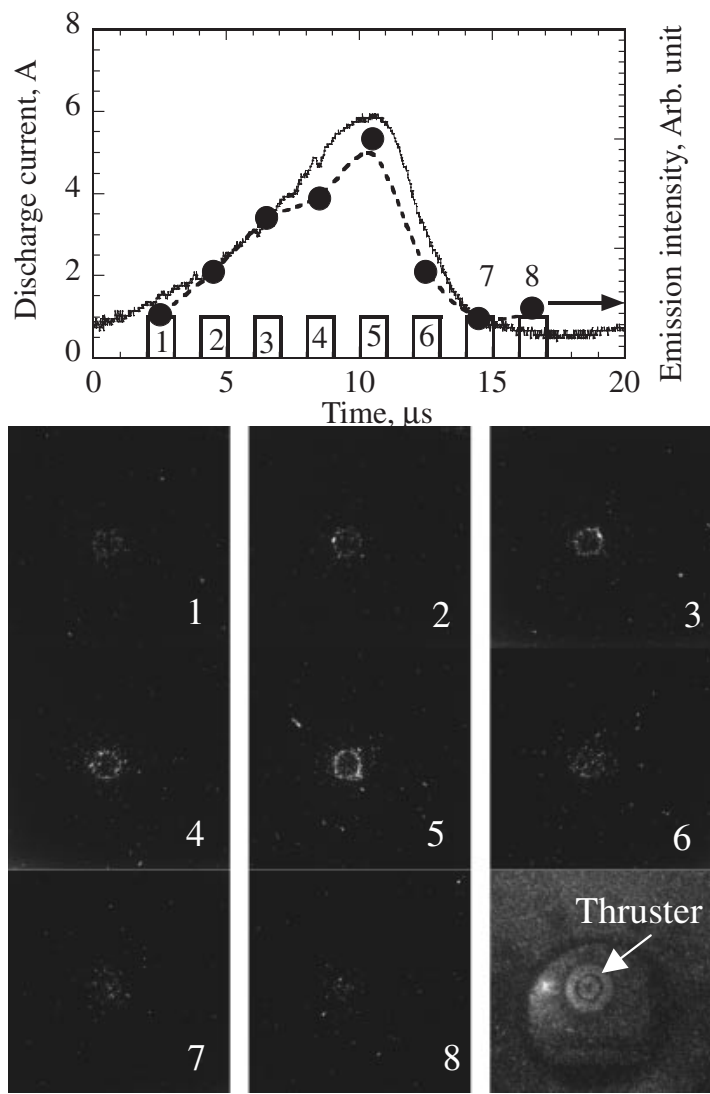

Fig. 5. Oscillation behavior of Xe II.

of continuity for the electron. The continuity for the ion was not used ${ }^{16)}$ because the diffusion rate of the plasma is controlled by the slower species, which is the electron in the Hall thruster where it is trapped by the magnetic field.

The equation of continuity for the neutral atom is expressed as follows:

$$
\frac{\partial N_{\mathrm{n}}}{\partial t}+\nabla\left(N_{\mathrm{n}} V_{\mathrm{n}}\right)=-\left\langle\sigma_{\mathrm{di}} v_{\mathrm{e}}\right\rangle_{T_{\mathrm{e}}} N_{\mathrm{n}} N_{\mathrm{e}}
$$

The equation of continuity for the electron is expressed as follows:

$$
\frac{\partial N_{\mathrm{e}}}{\partial t}+\nabla\left(N_{\mathrm{e}} V_{\mathrm{e}}\right)=\left\langle\sigma_{\mathrm{di}} v_{\mathrm{e}}\right\rangle_{T_{\mathrm{e}}} N_{\mathrm{n}} N_{\mathrm{e}}
$$

Equations (2) and (3) are integrated over the entire ionization zone because the plasma oscillates monolithically in this zone (Fig. 4).

$$
\begin{aligned}
& \int_{V} \frac{\partial N_{\mathrm{n}}}{\partial t} \mathrm{~d} V+\int_{S} N_{\mathrm{n}} V_{\mathrm{n}} \mathrm{d} S=\int_{V}-\left\langle\sigma_{\mathrm{di}} v_{\mathrm{e}}\right\rangle_{T_{\mathrm{e}}} N_{\mathrm{n}} N_{\mathrm{e}} \mathrm{d} V \\
& \int_{V} \frac{\partial N_{\mathrm{e}}}{\partial t} \mathrm{~d} V+\int_{S} N_{\mathrm{e}} V_{\mathrm{e}} \mathrm{d} S=\int_{V}\left\langle\sigma_{\mathrm{di}} v_{\mathrm{e}}\right\rangle_{T_{\mathrm{e}}} N_{\mathrm{n}} N_{\mathrm{e}} \mathrm{d} V
\end{aligned}
$$

Linearization was used to solve these equations analytically. The phase velocity of the perturbation of neutral atoms was propagated as its axial velocity.

$$
N_{\mathrm{n}}=N_{\mathrm{n}}+n_{\mathrm{n}} \exp \left[-i\left(\omega t-k_{\mathrm{n}} z\right)\right]
$$

Where, $k_{\mathrm{n}}=C_{\mathrm{n}}+i 1 / \lambda_{\mathrm{ne}}$, and $C_{\mathrm{n}} \approx \operatorname{Re}[\omega] / 2 \pi V_{\mathrm{n}}$
A unique feature of the present model is that a disturbance in the plasma oscillates monolithically in the entire ionization zone, although Baranov assumed that a perturbation of plasma propagates with the same phase velocity as that of a perturbation of neutral atoms. ${ }^{13)}$

$$
N_{\mathrm{e}}=N_{\mathrm{e}}+n_{\mathrm{e}} \exp [-i \omega t]
$$

For simplicity, $\left\langle\sigma_{\mathrm{di}} v_{\mathrm{e}}\right\rangle_{T_{\mathrm{e}}}, N_{\mathrm{n}}$ and $N_{\mathrm{e}}$ are considered as

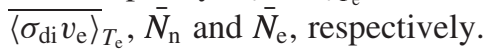

$$
\begin{aligned}
{\overline{\left\langle\sigma_{\mathrm{di}} v_{\mathrm{e}}\right\rangle_{T_{\mathrm{e}}}}}=\frac{1}{L} \int_{0}^{L}\left\langle\sigma_{\mathrm{di}} v_{\mathrm{e}}\right\rangle_{T_{\mathrm{e}}} \mathrm{d} z \\
\bar{N}_{\mathrm{n}}=\frac{1}{L} \int_{0}^{L} N_{\mathrm{n}} \mathrm{d} z, \bar{N}_{\mathrm{e}}=\frac{1}{L} \int_{0}^{L} N_{\mathrm{e}} \mathrm{d} z
\end{aligned}
$$

If an electron moves to the anode with classical diffusion, ${ }^{21)}$ the electron velocity is assumed to oscillate, because electron velocity is proportional to the neutral atom density. The electron velocity is expressed as follows:

$$
\begin{aligned}
V_{\mathrm{e}}=V_{\mathrm{e}}+v_{\mathrm{e}} & =-\frac{m_{\mathrm{e}}\left\langle\sigma_{\mathrm{T}} v_{\mathrm{e}}\right\rangle_{\mathrm{en}}}{e B^{2}}\left(E+\frac{k T_{\mathrm{e}}}{e} \frac{\nabla N_{\mathrm{e}}}{N_{\mathrm{e}}}\right)\left(N_{\mathrm{n}}+n_{\mathrm{n}}\right) \\
& \equiv f(z)\left(N_{\mathrm{n}}+n_{\mathrm{n}}\right) .
\end{aligned}
$$

Substituting Eqs. (5) and (6) into Eq. (4), the dispersion relation is written as:

$$
\begin{aligned}
\omega^{2} & +\left\{i \overline{\left\langle\sigma_{\mathrm{di}} v_{\mathrm{e}}\right\rangle_{T_{\mathrm{e}}}}\left(\bar{N}_{\mathrm{e}}-\bar{N}_{\mathrm{n}}\right)+i \frac{V_{\mathrm{e} 1}-r_{\mathrm{S}} V_{\mathrm{e} 0}}{L}-G V_{\mathrm{n}}\right\} \omega \\
& +i G\left(\overline{\left\langle\sigma_{\mathrm{di}} v_{\mathrm{e}}\right\rangle_{T_{\mathrm{e}}}} \bar{N}_{\mathrm{n}} V_{\mathrm{n}}-\frac{V_{\mathrm{e} 1}-r_{\mathrm{S}} V_{\mathrm{e} 0}}{L} V_{\mathrm{n}}\right. \\
& \left.+{\overline{\left\langle\sigma_{\mathrm{di}} v_{\mathrm{e}}\right\rangle_{T_{\mathrm{e}}}}}_{\bar{N}_{n}} f_{1} N_{\mathrm{e} 1}\right) \\
& -{\overline{\left\langle\sigma_{\mathrm{di}} v_{\mathrm{e}}\right\rangle_{T_{\mathrm{e}}}}}_{\bar{N}_{\mathrm{e}}} \frac{V_{\mathrm{e} 1}-r_{\mathrm{S}} V_{\mathrm{e} 0}}{L}=0
\end{aligned}
$$

where $G=\frac{k_{\mathrm{n}} \times \exp \left[i k_{\mathrm{n}} L\right]}{\exp \left[i k_{\mathrm{n}} L\right]-1}$ and $r_{\mathrm{S}}=\frac{S_{0}}{S_{1}}$

After solving the above dispersion relation, the frequency of this oscillation, $\operatorname{Re}[\omega]$, is written as follows:

$$
\begin{aligned}
f_{\mathrm{a}} & =\frac{1}{2 \pi} \operatorname{Re}[\omega] \\
& =\frac{1}{2 \pi}\left(\frac{1}{2}\left(-a+\operatorname{Re}\left[\sqrt{(a+i b)^{2}-4(c+i d)}\right]\right)\right)
\end{aligned}
$$

The stability condition of this oscillation, $\operatorname{Im}[\omega]<0$ is written as follows:

$$
\begin{gathered}
b>0 \\
b^{2} c-a b d+d^{2}<0
\end{gathered}
$$

where

$$
\begin{aligned}
& a=-\operatorname{Re}[\mathrm{G}] \times V_{\mathrm{n}} \\
& b=\frac{V_{\mathrm{e} 1}-r_{\mathrm{S}} V_{\mathrm{e} 0}}{L}-{\overline{\left\langle\sigma_{\mathrm{di}} v_{\mathrm{e}}\right\rangle_{T_{\mathrm{e}}}}}_{N_{\mathrm{n}}}+{\overline{\left\langle\sigma_{\mathrm{di}} v_{\mathrm{e}}\right\rangle_{T_{\mathrm{e}}}}}_{\bar{N}_{\mathrm{e}}}-\operatorname{Im}[G] \times V_{\mathrm{n}} \\
& c=-\operatorname{Im}[\mathrm{G}] \times\left({\overline{\left\langle\sigma_{\mathrm{di}} v_{\mathrm{e}}\right\rangle_{T_{\mathrm{e}}}}}_{\bar{N}_{\mathrm{n}}} V_{\mathrm{n}}-\frac{V_{\mathrm{e} 1}-r_{\mathrm{S}} V_{\mathrm{e} 0}}{L} V_{\mathrm{n}}\right.
\end{aligned}
$$

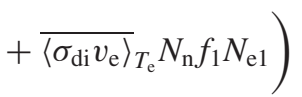




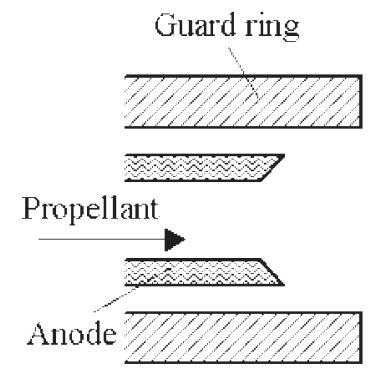

(a)
Guard ring
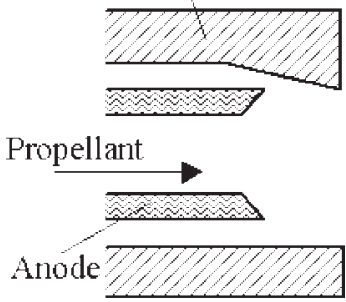

(b)
Fig. 6. Images of various guard rings. (a) parallel, (b) convergent.

$$
\begin{aligned}
& -{\overline{\left\langle\sigma_{\mathrm{di}} v_{\mathrm{e}}\right\rangle_{T_{\mathrm{e}}}}}_{\bar{N}_{\mathrm{e}} \frac{V_{\mathrm{e} 1}-r_{\mathrm{S}} V_{\mathrm{e} 0}}{L}}^{d=} \operatorname{Re}[\mathrm{G}] \times\left(\overline{\left\langle\sigma_{\mathrm{di}} v_{\mathrm{e}}\right\rangle_{T_{\mathrm{e}}}} \bar{N}_{\mathrm{n}} V_{\mathrm{n}}-\frac{V_{\mathrm{e} 1}-r_{\mathrm{S}} V_{\mathrm{e} 0}}{L} V_{\mathrm{n}}\right. \\
& +{\overline{\left\langle\sigma_{\mathrm{di}} v_{\mathrm{e}}\right\rangle_{T_{\mathrm{e}}}}}_{\left.N_{\mathrm{n}} f_{1} N_{\mathrm{e} 1}\right)}
\end{aligned}
$$

\subsection{Discharge current oscillation suppression method}

The stable operational condition predicted by this model can be written simply as follows:

$$
S_{1} V_{\mathrm{e} 1}-S_{0} V_{\mathrm{e} 0}-{\overline{\left\langle\sigma_{\mathrm{di}} v_{\mathrm{e}}\right\rangle_{T_{\mathrm{e}}}}}_{\bar{N}_{\mathrm{n}}} S L>0
$$

The left side of Eq. (13) represents the momentum transfer corresponding to the plasma fluctuations. Thus, the oscillations decay if the left side of Eq. (13) is positive. In other words, this model shows that the convergent acceleration channel configuration could allow the oscillation to be suppressed because it improves propellant utilization, resulting in a change in $V_{\mathrm{e} 1}$ for classical diffusion. Hence, $\Delta$ was measured for various guard rings (Fig. 6). The inner and outer diameters of the guard rings of the convergent type are $48 \mathrm{~mm}$ and $70 \mathrm{~mm}$, respectively. Figure 7(a) shows the experimental results of $\Delta$ with the parallel acceleration channel and Fig. 7(b) shows $\Delta$ with the convergent acceleration channel. The stable operational condition range of the convergent type is greater than that of the parallel type. Consequently, the discharge current oscillations in the Hall thruster were suppressed successfully. Moreover, propellant utilization using the convergent configuration is larger than that of the parallel type. As a result, the thrust efficiency of the convergent configuration is higher than that of the parallel one. ${ }^{22)}$

\section{Summary}

Discharge current oscillation characteristics at a frequency range of $10-100 \mathrm{kHz}$ were investigated using a $1-\mathrm{kW}$ class, anode layer type Hall thruster. The plasma behavior in the acceleration channel was investigated by a high-speed camera to clarify this oscillation mechanism. The emission intensity oscillated monolithically in the acceleration channel with the same period as that of the discharge current oscillation, and its intensity was proportional to the discharge

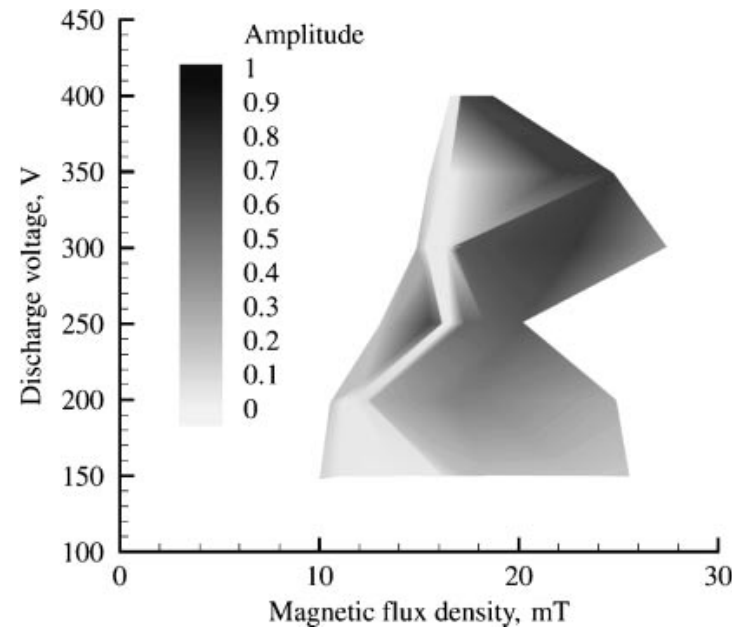

(a)

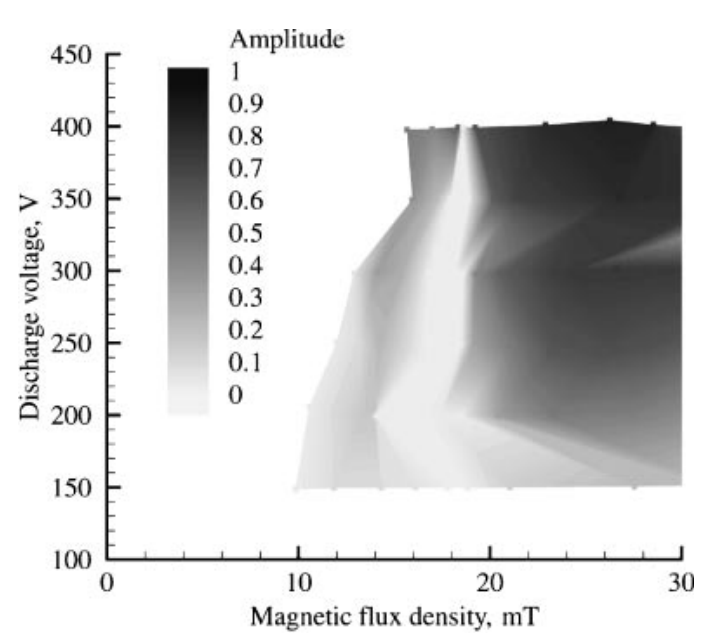

(b)

Fig. 7. Oscillation amplitude for various guard rings. (a) parallel, (b) convergent. $\dot{m}=2.72 \mathrm{mg} / \mathrm{s}$.

current. These results indicate that this oscillation is derived from the fluctuation of the plasma density in the acceleration channel, i.e., this oscillation is caused by ionization instability and the number density of ions is oscillated monolithically in the entire acceleration channel. Additionally, the oscillation amplitude sensitively changed with magnetic flux density. These results show that the oscillation is dominated by electron mobility rather than by ion mobility. Therefore, an oscillation model considering the electron dynamics was proposed. This model shows that the acceleration channel configuration affects the oscillation. In other words, the convergent acceleration channel configuration leads to an extension in the stable operational range, because it leads to an increase in propellant utilization, resulting in an increase in the momentum transfer corresponding to plasma fluctuation; this increases the stable operational range. Consequently, the stable operational range was investigated for two acceleration channel configurations-parallel and convergent. As shown by this model, the stable operational range was successfully extended by use of the convergent configuration. 


\section{Acknowledgments}

The present work was supported by a Grant-in-Aid for Scientific Research (S), No. 16106012, sponsored by the Ministry of Education, Culture, Sports, Science and Technology, Japan.

\section{References}

1) Saccoccia, G.: Introduction to the European Activities in Electric Propulsion, IEPC Paper 03-341, 2003.

2) Blandino, J.: The Year in Review, Electric Propulsion, Aerospace Am., December (2003), pp. 60-61.

3) Kaufman, H. R.: Technology of Closed-Drift Thrusters, AIAA J., 23 (1985), pp. 78-86.

4) Choueiri, E. Y.: Fundamental Difference between the Two Hall Thruster Variants, Phys. Plasmas, 8 (2001), pp. 5025-5033.

5) Morozov, A. I., Esipchuk, Yu. V., Tilinin, G. N., Trofimov, A. V., Sharov, Yu. A. and Shchepkin, G. Ya.: Plasma Accelerator with Closed Electron Drift and Extended Acceleration Zone, Sov. Phys.-Tech. Phys., 17 (1972), pp. 38-45.

6) Kim, V.: Main Physical Feature and Processes Determining the Performance of Stationary Plasma Thrusters, J. Propul. Power, 14 (1998), pp. 736-743.

7) Zharinov, A. V. and Popov, Yu. S.: Acceleration of Plasma by a Closed Hall Current, Sov. Phys.-Tech. Phys., 12 (1967), pp. 208-211.

8) Popov, Yu. S. and Zolotaikin, Yu. M.: Effect of Anomalous Conductivity on the Structure of the Anode Sheath in a Hall Current Ion Source, Sov. J. Plasma Phys., 3 (1977), pp. 210-213.

9) Garner, C. E., Brophy, J. R., Polk, J. E., Semenkin, A. V., Garlusha, V. I., Tverdokhlebov, S. O. and Marrese, C.: Experimental Evaluation of Russian Anode Layer Thrusters, AIAA Paper 94-3010, 1994.

10) Garner, C. E., Brophy, J. R., Polk, J. E., Semenkin, A. V., Garlusha, V. I., Tverdokhlebov, S. O. and Marrese, C.: Experimental Evaluation of
Russian Anode Layer Thrusters, AIAA Paper 94-3010, 1994.

11) Zhurin, V. V., Kaufman, H. R. and Robinson, R. S.: Physics of Closed Drift Thrusters, Plasma Sources Sci. T., 8 (1999), R1-R20.

12) Solodukhin, A. E. and Semenkin, A. V.: Study of Discharge Channel Erosion in Multimode Anode Layer Thruster, IEPC Paper 2003-0204, 2003.

13) Baranov, V. I., Nazarenko, Yu. S., Petrosov, V. A., Vasin, A. I. and Yashonov, Yu. M.: Theory of Oscillations and Conductivity for Hall Thruster, AIAA Paper 96-3192, 1996.

14) Boeuf, J. P. and Garrigues, L.: Low Frequency Oscillation in a Stationary Plasma Thruster, J. Appl. Phys., 84 (1998), pp. 3541-3554.

15) Komurasaki, K. and Kusamoto, D.: Optical Measurement of Plasma Oscillations in a Hall Thruster, Trans. Jpn. Soc. Aeronaut. Space Sci., 42 (1999), pp. 203-208.

16) Fife, J. M., Martinez-Sanchez, M. and Szabo, J.: A Numerical Study of Low-frequency Discharge oscillations in Hall Thrusters, AIAA Paper 97-3052, 1997.

17) Tilinin, G. N.: High-Frequency Plasma Waves in a Hall Accelerator with an Extended Acceleration Zone, Sov. Phys.-Tech. Phys., 22 (1977), pp. 974-978.

18) Choueiri, E. Y.: Plasma Oscillations in Hall Thrusters, Phys. Plasmas, 8 (2001), pp. 1411-1426.

19) Furukawa, T., Miyasaka, T. and Fujiwara, T.: Control of Low-Frequency Oscillation in a Hall thruster, Trans. Jpn. Soc. Aeronaut. Space Sci., 44 (2001), pp. 164-170.

20) Yamamoto, N., Komurasaki, K. and Arakawa, Y.: Operating Characteristics of an Anode Layer Type Hall Thruster, J. Jpn. Soc. Aeronaut. Space Sci., 51 (2003), pp. 492-497 (in Japanese).

21) Yamamoto, N., Komurasaki, K. and Arakawa, Y.: A Suppression Method of Discharge Current Oscillations in a Hall Thruster, AIAA Paper 2004-4100, 2004.

22) Yamamoto, N.: Discharge Current Oscillation in Hall Thrusters, Ph.D. Diss., The Univ. of Tokyo, 2004 (in Japanese). 